\title{
THE RAPIDITY OF CONVERGENCE OF THE HERMITE- FE JER APPROXIMATION TO FUNCTIONS OF ONE OR SEVERAL VARIABLES
}

O. SHISHA AND B. MOND

1. The Hermite-Fejér polynomials $H_{n}(f, x)$ are important approximating polynomials to a given real function $f(x)$ defined on $[-1,1]$. For every positive integer $n$,

$$
H_{n}(f, x) \equiv \sum_{k=1}^{n} f\left(x_{k}^{(n)}\right) A_{k}^{(n)}(x)
$$

where

$$
\begin{array}{r}
A_{k}^{(n)}(x) \equiv\left(1-x x_{k}^{(n)}\right)\left[T_{n}(x) /\left\{n\left(x-x_{k}^{(n)}\right)\right\}\right]^{2} \quad(k=1,2, \cdots, n), \\
T_{n}(x) \equiv 2^{n-1} \prod_{k=1}^{n}\left(x-x_{k}^{(n)}\right), \quad x_{k}^{(n)}=\cos \left(\frac{2 k-1}{2 n} \pi\right) \\
(k=1,2, \cdots, n) .
\end{array}
$$

For $n=1,2, \cdots, T_{n}(x)$ (the $n$th degree Tchebycheff polynomial of the first kind) satisfies $T_{n}(\cos \theta) \equiv \cos (n \theta)$, and we also have

$$
A_{k}^{(n)}(x) \geqq 0 \quad \text { for } k=1,2, \cdots, n \text { and every } x \in[-1,1],
$$

(3) $\sum_{k=1}^{n} A_{k}^{(n)}(x) \equiv 1$,

(4) $\quad H_{n}\left(f, x_{k}^{(n)}\right)=f\left(x_{k}^{(n)}\right) \quad(k=1,2, \cdots, n)$,

(5) $\quad H_{n}^{\prime}\left(f, x_{k}^{(n)}\right)=0 \quad(k=1,2, \cdots, n)$.

2. Suppose $f$ is a real function, continuous in $[-1,1]$. Then a classical result of Fejér [1] states that $H_{n}(f, x)$ converges uniformly to $f(x)$ on $[-1,1]$. As to the rapidity of convergence, E. Moldovan published in [2] the estimate $\max _{-1 \leq x \leq 1}\left|f(x)-H_{n}(f, x)\right| \leqq 2 \pi \omega\left(n^{-1} \log n\right)$, where $\omega$ is the modulus of continuity of $f$ in $[-1,1]$.

3. One of our purposes in this paper is to construct an analog of the polynomial (1) for functions $f$ of several variables and to study the corresponding rapidity of convergence. Since the Romanian paper

Presented to the Society, April 13, 1965 under the title Hermite-Fejer polynomials for functions of several variables; received by the editors November 18, 1964. 
[2] is inaccessible to many readers and is also wanting, we shall prove in Theorem 2 a result which is essentially the same as that published by Moldovan. Theorems 1 and 2 will be used to obtain Theorems 3 and 4 concerning functions of several variables.

4. THEOREM 1. Let $f$ be a real function satisfying throughout $[-1,1]$

$$
|f(v)-f(u)| \leqq \lambda|v-u|
$$

where $\lambda$ is a positive constant. Then for $n=1,2, \cdots$ and every $x \in[-1,1]$,

$$
\left|f(x)-B_{n}(f, x)\right|<4 \lambda \pi n^{-1}(\alpha+\log n),
$$

where $\alpha=\frac{1}{2}+C-\log 2=0.384 \cdots, C$ being Euler's constant.

Proof. Let $n$ be a positive integer and let $-1 \leqq x \leqq 1$. We shall prove (6). Let $x=\cos \theta(0 \leqq \theta \leqq \pi)$, and let $\theta_{k}=((2 k-1) / 2 n) \pi$ $(k=1,2, \cdots, n)$. Since by (4), $H_{n}\left(f, \cos \theta_{k}\right)=f\left(\cos \theta_{k}\right)$ for $k=1,2$, $\cdots, n$, we may assume $\theta \neq \theta_{k}, k=1,2, \cdots, n$. By (3), (1), and (2) $\left|f(x)-B_{n}(f, x)\right|$

$$
=\left|\sum_{k=1}^{n}\left[f(x)-f\left(x_{k}^{(n)}\right)\right] A_{k}^{(n)}(x)\right| \leqq \sum_{k=1}^{n}\left|f(x)-f\left(x_{k}^{(n)}\right)\right| A_{k}^{(n)}(x)
$$$$
=\sum_{k=1}^{n}\left|f(x)-f\left(x_{k}^{(n)}\right)\right|\left(1-x x_{k}^{(n)}\right)\left[T_{n}(x) /\left\{n\left(x-x_{k}^{(n)}\right)\right\}\right]^{2}
$$$$
\leqq \lambda \sum_{k=1}^{n}\left|x-x_{k}^{(n)}\right|\left(1-x x_{k}^{(n)}\right)\left[T_{n}(x) /\left\{n\left(x-x_{k}^{(n)}\right)\right\}\right]^{2}
$$$$
=\lambda n^{-2} \sum_{k=1}^{n}\left|\cos \theta-\cos \theta_{k}\right|\left(1-\cos \theta \cos \theta_{k}\right)\left[\cos (n \theta) /\left(\cos \theta-\cos \theta_{k}\right)\right]^{2}
$$$$
<\lambda n^{-2} \sum_{k=1}^{n}\left|\theta-\theta_{k}\right|\left(1-\cos \theta \cos \theta_{k}\right)\left[\cos (n \theta) /\left(\cos \theta-\cos \theta_{k}\right)\right]^{2}
$$

$$
\begin{aligned}
\leqq & \lambda n^{-2} \sum_{k=1}^{n}\left|\theta-\theta_{k}\right|\left[1-\cos \theta \cos \theta_{k}+\sin \theta \sin \theta_{k}\right] \\
& \cdot\left[\left(\cos (n \theta)-\cos \left(n \theta_{k}\right)\right) /\left(\cos \theta-\cos \theta_{k}\right)\right]^{2} \\
= & \lambda n^{-2} \sum_{k=1}^{n}\left|\theta-\theta_{k}\right| 2 \sin ^{2}\left\{\left(\theta+\theta_{k}\right) / 2\right\} \sin ^{2}\left\{n\left(\theta+\theta_{k}\right) / 2\right\} \\
& \cdot \sin ^{2}\left\{n\left(\theta-\theta_{k}\right) / 2\right\} \sin ^{-2}\left\{\left(\theta+\theta_{k}\right) / 2\right\} \sin ^{-2}\left\{\left(\theta-\theta_{k}\right) / 2\right\} \\
< & 2 \lambda n^{-2} \sum_{k=1}^{n}\left|\theta-\theta_{k}\right|\left[\sin \left\{n\left(\theta-\theta_{k}\right) / 2\right\} / \sin \left\{\left(\theta-\theta_{k}\right) / 2\right\}\right]^{2} .
\end{aligned}
$$


Suppose $n \geqq 4$ and $\theta_{j}<\theta<\theta_{j+1}, 2 \leqq j \leqq n-2$. Since $|\sin (n y) / \sin y|$ $<n$ for every real $y \neq 0, \pm \pi, \pm 2 \pi, \cdots$, therefore

$$
\begin{aligned}
\left|\theta-\theta_{j}\right|\left[\sin \left\{n\left(\theta-\theta_{j}\right) / 2\right\} / \sin \left\{\left(\theta-\theta_{j}\right) / 2\right\}\right]^{2} \\
\quad+\left|\theta-\theta_{j+1}\right|\left[\sin \left\{n\left(\theta-\theta_{j+1}\right) / 2\right\} / \sin \left\{\left(\theta-\theta_{j+1}\right) / 2\right\}\right]^{2} \\
<\left|\theta-\theta_{j}\right| n^{2}+\left|\theta-\theta_{j+1}\right| n^{2}=n \pi .
\end{aligned}
$$

Since $y / \sin (y / 2)$ is strictly increasing in $(0, \pi)$, we have $y / \sin (y / 2)$ $<\pi / \sin (\pi / 2)=\pi$ whenever $0<y<\pi$. For such $y$ one has

$$
y[\sin (n y / 2) / \sin (y / 2)]^{2}=y^{-1}[y / \sin (y / 2)]^{2} \sin ^{2}(n y / 2)<\pi^{2} / y .
$$

For $k=1,2, \cdots, j-1, \theta-\theta_{k}>(j-k) \pi / n$, and so $\left(\theta-\theta_{k}\right)\left[\sin \left\{n\left(\theta-\theta_{k}\right) / 2\right\} / \sin \left\{\left(\theta-\theta_{k}\right) / 2\right\}\right]^{2}<\pi^{2} /\left(\theta-\theta_{k}\right)<n \pi /(j-k)$.

Consequently,

$$
\begin{gathered}
\sum_{k=1}^{+1}\left|\theta-\theta_{k}\right|\left[\sin \left\{n\left(\theta-\theta_{k}\right) / 2\right\} / \sin \left\{\left(\theta-\theta_{k}\right) / 2\right\}\right]^{2} \\
<n \pi \sum_{k=1}^{j-1}(j-k)^{-1}=n \pi \sum_{k=1}^{j-1} k^{-1} .
\end{gathered}
$$

Similarly,

(9)

$$
\begin{gathered}
\sum_{k=j+2}^{n}\left|\theta-\theta_{k}\right|\left[\sin \left\{n\left(\theta-\theta_{k}\right) / 2\right\} / \sin \left\{\left(\theta-\theta_{k}\right) / 2\right\}\right]^{2} \\
<n \pi \sum_{k=j+2}^{n}[k-(j+1)]^{-1}=n \pi \sum_{k=1}^{n-1-j} k^{-1}
\end{gathered}
$$

From (8), (7) and (9) we obtain

$$
\begin{aligned}
& \sum_{k=1}^{n}\left|\theta-\theta_{k}\right|\left[\sin \left\{n\left(\theta-\theta_{k}\right) / 2\right\} / \sin \left\{\left(\theta-\theta_{k}\right) / 2\right\}\right]^{2} \\
& <n \pi\left(1+\sum_{k=1}^{j-1} k^{-1}+\sum_{k=1}^{n-1-j} k^{-1}\right)
\end{aligned}
$$

and therefore

$$
\left|f(x)-H_{n}(f, x)\right|<2 \lambda \pi n^{-1}\left(1+\sum_{k=1}^{+1} k^{-1}+\sum_{k=1}^{n-1-j} k^{-1}\right) .
$$

If $n$ is even, then 


$$
\begin{aligned}
\sum_{k=1}^{j-1} k^{-1}+\sum_{k=1}^{n-1-j} k^{-1} & \leqq \max _{1 \leq q \leq n-3}\left(\sum_{k=1}^{q} k^{-1}+\sum_{k=1}^{n-2-q} k^{-1}\right) \\
& =2\left[1+2^{-1}+\cdots+\{(n-2) / 2\}^{-1}\right] .
\end{aligned}
$$

If $n$ is odd, then

$$
\begin{aligned}
\sum_{k=1}^{j-1} k^{-1}+\sum_{k=1}^{n-1-j} k^{-1} & \leqq \max _{1 \leqq q \leqq n-3}\left(\sum_{k=1}^{q} k^{-1}+\sum_{k=1}^{n-2-q} k^{-1}\right) \\
& =2\left[1+2^{-1}+\cdots+\{(n-3) / 2\}^{-1}\right]+\{(n-1) / 2\}^{-1} .
\end{aligned}
$$

Thus

$$
\begin{array}{r}
\left|f(x)-B_{n}(f, x)\right|<2 \lambda \pi n^{-1}\left(1+2\left[1+2^{-1}+\cdots+\{(n-2) / 2\}^{-1}\right]\right) \\
\text { if } n \text { is even, } \\
\left|f(x)-B_{n}(f, x)\right|<2 \lambda \pi n^{-1}\left(1+2\left[1+2^{-1}+\cdots+\{(n-3) / 2\}^{-1}\right.\right. \\
\left.\left.+(n-1)^{-1}\right]\right) \quad \text { if } n \text { is odd. }
\end{array}
$$

One can show similarly, that (11) (with $n \geqq 4$ ) is true for every other position of $\theta$ in $[0, \pi]$.

Since $\left(\sum_{k=1}^{q-1} k^{-1}\right)-\log q<C$ for $q=2,3,4, \cdots$, the desired inequality (6) follows from (11) for $n$ even and $\geqq 4$.

Consider the sequence

$$
a_{q} \equiv\left(\sum_{k=1}^{q-1} k^{-1}\right)+(2 q)^{-1}-\log [(2 q+1) / 2] \quad(q=2,3,4, \cdots) .
$$

For every $q \geqq 2$ we have

$$
\begin{aligned}
a_{q+1}-a_{q}= & \frac{1}{2}\left[q^{-1}+(q+1)^{-1}\right]-\log [1+2 /(2 q+1)] \\
& >\frac{1}{2}\left[q^{-1}+(q+1)^{-1}\right]-2 /(2 q+1)>0 .
\end{aligned}
$$

Since $\lim _{q \rightarrow \infty} a_{q}=C$, we have $a_{q}<C(q=2,3,4, \cdots)$.

Suppose $n$ is odd and $>4$. Setting $q=(n-1) / 2$ we have $C>a_{q}$ $=1+2^{-1}+\cdots+\{(n-3) / 2\}^{-1}+(n-1)^{-1}-\log (n / 2)$, and (6) follows from (11).

Finally one can verify (6) for $n=1,2,3$ by the same sort of calculations which led to (10).

5. THEOREM 2. Let $f$ be a real function, defined and bounded on $[-1,1]$. For every $\delta \in[0,2]$ let

$$
\omega(\delta)=\sup _{-1 \leqq u \leq 0 \leq 1 ; 0-u \leq \delta}|f(v)-f(u)| .
$$


Then for $n=2,3,4, \cdots$ we have

$$
\sup _{-1 \leqq x \leqq 1}\left|f(x)-H_{n}(f, x)\right| \leqq\left[2+4 \pi+\epsilon_{n}\right] \omega\left(n^{-1} \log n\right)
$$

where $\epsilon_{n}$ depends on $n$ only and $\epsilon_{n} \rightarrow 0$ as $n \rightarrow \infty$.

Proof. Let $n$ be a positive integer. Let $x_{0}, x_{1}, \cdots, x_{N}(N \geqq 1)$ be reals with $-1=x_{0}<x_{1}<\cdots<x_{N}=1$. Let $f_{n}$ be the function with domain $[-1,1]$ such that $f_{n}\left(x_{k}\right)=f\left(x_{k}\right), k=0,1, \cdots, N$ and such that $f_{n}$ is linear in each $\left[x_{k-1}, x_{k}\right](k=1,2, \cdots, N)$. If $x_{k-1} \leqq u<v \leqq x_{k}$ for some $k$, then

$$
\begin{aligned}
\left|f_{n}(v)-f_{n}(u)\right| /(v-u) & =\left|f\left(x_{k}\right)-f\left(x_{k-1}\right)\right| /\left(x_{k}-x_{k-1}\right) \\
& \leqq \omega\left(x_{k}-x_{k-1}\right) /\left(x_{k}-x_{k-1}\right) \leqq \lambda
\end{aligned}
$$

where

$$
\lambda=\max _{1 \leqq k \leqq N}\left[\omega\left(x_{k}-x_{k-1}\right) /\left(x_{k}-x_{k-1}\right)\right] .
$$

Therefore $\left|f_{n}(v)-f_{n}(u)\right| \leqq \lambda(v-u)$ whenever $-1 \leqq u<v \leqq 1$. By Theorem 1 we have throughout $[-1,1]$,

$$
\left|f_{n}(x)-B_{n}\left(f_{n}, x\right)\right| \leqq 4 \lambda \pi n^{-1}(\alpha+\log n) .
$$

One easily verifies that throughout $[-1,1]$,

$$
\left|f(x)-f_{n}(x)\right| \leqq \mu
$$

where $\mu=\max _{1 \leq k \leq N} \omega\left(x_{k}-x_{k-1}\right)$. Therefore, by (1), (2) and (3) we have throughout $[-1,1]$,

$$
\left|H_{n}(f, x)-B_{n}\left(f_{n}, x\right)\right| \leqq \sum_{k=1}^{n}\left|f\left(x_{k}^{(n)}\right)-f_{n}\left(x_{k}^{(n)}\right)\right| A_{k}^{(n)}(x) \leqq \mu .
$$

From (12), (13) and (14) we get

$$
\sup _{-1 \leqq x \leqq 1}\left|f(x)-H_{n}(f, x)\right| \leqq 2 \mu+4 \lambda \pi n^{-1}(\alpha+\log n)
$$

and so

$$
\begin{aligned}
& \sup _{-1 \leqq x \leqq 1}\left|f(x)-H_{n}(f, x)\right| \\
& \leqq \inf _{-1=x_{0}<x_{1} \cdots<x_{N}=1 ; N-1,2,3, \ldots}\left\{2 \max _{1 \leqq k \leqq N} \omega\left(x_{k}-x_{k-1}\right)\right. \\
& \left.\quad+4 \max _{1 \leqq k \leqq N}\left[\omega\left(x_{k}-x_{k-1}\right) /\left(x_{k}-x_{k-1}\right)\right] \pi n^{-1}(\alpha+\log n)\right\} .
\end{aligned}
$$


If we take in particular for some positive integer $N, x_{k}=-1$ $+(2 k / N)(k=0,1, \cdots, N)$, then from (15) we obtain

$$
\sup _{-1 \leqq x \leqq 1}\left|f(x)-B_{n}(f, x)\right| \leqq 2 \omega(2 / N)\left[1+\pi N n^{-1}(\alpha+\log n)\right] .
$$

Thus

$\sup _{-1 \leqq x \leqq 1}\left|f(x)-B_{n}(f, x)\right| \leqq \inf _{N=1,2, \ldots}\left\{2 \omega(2 / N)\left[1+\pi N n^{-1}(\alpha+\log n)\right]\right\}$.

Suppose $n \geqq 2$, and let $N$ be the integral part of $1+(2 n / \log n)$. Then (16) yields

$$
\sup _{-1 \leqq x \leqq 1}\left|f(x)-H_{n}(f, x)\right| \leqq\left[2+4 \pi+\epsilon_{n}\right] \omega\left(n^{-1} \log n\right),
$$

where $\epsilon_{n}=2 \pi n^{-1}[\alpha+\log n+(2 \alpha n / \log n)]$.

6. Consider now a real function $f\left(x_{1}, x_{2}, \cdots, x_{p}\right)$ defined for $-1 \leqq x_{k} \leqq 1, k=1,2, \cdots, p$. Let $n_{1}, n_{2}, \cdots, n_{p}$ be positive integers and set

$$
\begin{aligned}
& H_{n_{1}}, \cdots, n_{p} \\
& \quad \equiv \sum_{h_{1}=1}^{n_{1}} \cdots \sum_{h_{p}=1}^{n_{p}} f\left(x_{h_{1}}^{\left(n_{1}\right)}, \cdots, x_{h_{p}}^{\left(n_{p}\right)}\right) A_{h_{1}}^{\left(n_{1}\right)}\left(x_{1}\right) \cdots A_{h_{p}}^{\left(n_{p}\right)}\left(x_{p}\right) .
\end{aligned}
$$

The polynomial (18), which reduces to an Hermite-Fejér polynomial when $p=1$, has properties analogous to (4) and (5). Thus, for $k=1,2, \cdots, p$, let $j_{k}$ be a positive integer $\leqq n_{k}$. Then a repeated application of (4) yields easily

$$
H_{n_{1}, n_{2}}, \cdots, n_{p}\left(f, x_{j_{1}}^{\left(n_{1}\right)}, \cdots, x_{j_{p}}^{\left(n_{p}\right)}\right)=f\left(x_{j_{1}}^{\left(n_{1}\right)}, \cdots, x_{j_{p}}^{\left(n_{p}\right)}\right) .
$$

Also, if $p \geqq 2$, and if $1 \leqq k \leqq p, 1 \leqq j \leqq n_{k}$, then

$$
\left(\frac{\partial}{\partial x_{k}} B_{n_{1}, n_{2}}, \cdots, n_{p}\left(f, x_{1}, \cdots, x_{p}\right)\right)_{x_{k}=x_{j}^{\left(n_{k}\right)}} \equiv 0 .
$$

Indeed, the left-hand side of (19) is identically equal to

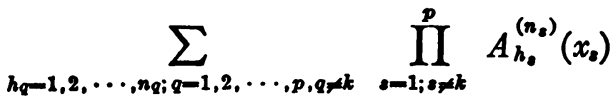

$$
\begin{aligned}
& \cdot\left(\frac{d}{d x_{k}} \sum_{h_{k}=1}^{n_{k}} f\left(x_{h_{1}}^{\left(n_{1}\right)}, \cdots, x_{h_{p}}^{\left(n_{p}\right)}\right) A_{h_{k}}^{\left(n_{k}\right)}\left(x_{k}\right)\right)_{x_{k}=x_{j}\left(n_{k}\right)}
\end{aligned}
$$

which is $\equiv 0$ by (1) and (5). 
From (3) one gets

$$
\sum_{h_{1}=1}^{n_{1}} \cdots \sum_{h_{p}=1}^{n_{p}} A_{h_{1}}^{\left(n_{1}\right)}\left(x_{1}\right) \cdots A_{h_{p}}^{\left(n_{p}\right)}\left(x_{p}\right) \equiv 1 .
$$

Consequently, by virtue of (18), if $p \geqq 2$,

$$
\begin{aligned}
& f\left(x_{1}, \cdots, x_{p}\right)-H_{n_{1}, \cdots, n_{p}}\left(f, x_{1}, \cdots, x_{p}\right) \\
& \equiv \sum_{h_{1}=1}^{n_{1}} \cdots \sum_{h_{p}=1}^{n_{p}}\left[f\left(x_{1}, \cdots, x_{p}\right)-f\left(x_{h_{1}}^{\left(n_{1}\right)}, \cdots, x_{h_{p}}^{\left(n_{p}\right)}\right)\right] \\
& \cdot A_{h_{1}}^{\left(n_{1}\right)}\left(x_{1}\right) \cdots A_{h_{p}}^{\left(n_{p}\right)}\left(x_{p}\right) \\
& \equiv \sum_{h_{1}=1}^{n_{1}} \cdots \sum_{h_{p}=1}^{n_{p}} \sum_{r=1}^{p}\left[f\left(x_{h_{1}}^{\left(n_{1}\right)}, \cdots, x_{h_{r-1}}^{\left(n_{r-1}\right)}, x_{r}, \cdots, x_{p}\right)\right. \\
& \left.-f\left(x_{h_{1}}^{\left(n_{1}\right)}, \cdots, x_{h_{r}}^{\left(n_{r}\right)}, x_{r+1}, \cdots, x_{p}\right)\right] \prod_{e-1}^{p} A_{h_{\varepsilon}}^{\left(n_{\varepsilon}\right)}\left(x_{s}\right) \\
& \equiv \sum_{r=1}^{p} \sum_{h_{q-1,2}, \ldots, n q ; q=1,2, \ldots, p, q \neq r}\left\{\sum _ { h _ { r } = 1 } ^ { n _ { r } } \left[f\left(x_{h_{1}}^{\left(n_{1}\right)}, \cdots, x_{h_{r-1}}^{\left(n_{r-1}\right)}, x_{r}, \cdots, x_{p}\right)\right.\right. \\
& \left.\left.-f\left(x_{h_{1}}^{\left(n_{1}\right)}, \cdots, x_{h_{r}}^{\left(n_{r}\right)}, x_{r+1}, \cdots, x_{p}\right)\right] A_{h_{r}}^{\left(n_{r}\right)}\left(x_{r}\right)\right\} \prod_{\varepsilon=1 ; \varepsilon \neq r}^{p} A_{h_{s}}^{\left(n_{s}\right)}\left(x_{s}\right) .
\end{aligned}
$$

Here and below $f\left(x_{h_{1}}^{\left(n_{1}\right)}, \cdots, x_{h_{r-1}}^{\left(n_{r-1}\right)}, x_{r}, \cdots, x_{p}\right)$ means $f\left(x_{1}, \cdots, x_{p}\right)$ if $r=1$, and $f\left(x_{h_{1}}^{\left(n_{1}\right)}, \cdots, x_{h_{r}}^{\left(n_{r}\right)}, x_{r+1}, \cdots, x_{p}\right)$ means $f\left(x_{h_{1}}^{\left(n_{1}\right)}, \cdots, x_{h_{p}}^{\left(n_{p}\right)}\right)$ if $r=p$.

7. THEOREM 3. Let $f\left(x_{1}, x_{2}, \cdots, x_{p}\right)(p \geqq 2)$ be a real function defined for $-1 \leqq x_{k} \leqq 1, k=1,2, \cdots, p$. For $r=1,2, \cdots, p$, let $\lambda_{r}$ be $a$ positive constant such that

$$
\begin{array}{r}
\left|f\left(x_{1}, \cdots, x_{r-1}, v, x_{r+1}, \cdots, x_{p}\right)-f\left(x_{1}, \cdots, x_{r-1}, u, x_{r+1}, \cdots, x_{p}\right)\right| \\
\leqq \lambda_{r}|v-u|
\end{array}
$$

whenever the $x_{q}, u$, and $v$ are all in $[-1,1]$. Let $n_{1}, n_{2}, \cdots, n_{p}$ be positive integers. Then

$$
\begin{gathered}
\left|f\left(x_{1}, x_{2}, \cdots, x_{p}\right)-B_{n_{1}, n_{2}}, \cdots, n_{p}\left(f, x_{1}, x_{2}, \cdots, x_{p}\right)\right| \\
<\sum_{r=1}^{p} 4 \lambda_{r} \pi n_{r}^{-1}\left(\alpha+\log n_{r}\right)
\end{gathered}
$$

throughout the cube $-1 \leqq x_{k} \leqq 1, k=1,2, \cdots, p$.

Proof. Let $x_{1}, x_{2}, \cdots, x_{p}$ be points of $[-1,1]$. We shall prove (20). For $r=1,2, \cdots, p$, we have by (3), (1), and by Theorem 1 , 


$$
\begin{aligned}
& \mid \sum_{n_{r}=1}^{n_{r}}\left[f\left(x_{h_{1}}^{\left(n_{1}\right)}, \cdots, x_{h_{r-1}}^{\left(n_{r-1}\right)}, x_{r}, \cdots, x_{p}\right)\right. \\
& \left.\quad-f\left(x_{h_{1}}^{\left(n_{1}\right)}, \cdots, x_{h_{r}}^{\left(n_{r}\right)}, x_{r+1}, \cdots, x_{p}\right)\right] A_{h_{r}}^{\left(n_{r}\right)}\left(x_{r}\right) \mid \\
& \quad<4 \lambda_{r} \pi n_{r}^{-1}\left(\alpha+\log n_{r}\right) .
\end{aligned}
$$

Since for $r=1,2, \cdots, p$,

$$
\sum_{n_{q}=1,2, \ldots, n_{q} ; q=1,2, \ldots, p, q \neq r} \prod_{s=1 ; \& \neq r}^{p} A_{h_{q}}^{\left(n_{s}\right)}\left(x_{s}\right)=1
$$

and each summand is $\geqq 0$, therefore by the last paragraph of section 6. (20) holds.

Similarly, from Theorem 2 we obtain the following

TheOREM 4. Let $f\left(x_{1}, x_{2}, \cdots, x_{p}\right)(p \geqq 2)$ be a real function defined and bounded for $-1 \leqq x_{k} \leqq 1, k=1,2, \cdots, p$. For every $\delta \in[0,2]$ and every $r(=1,2, \cdots, p)$ let

$$
\begin{aligned}
\omega_{r}(\delta)=\sup \mid f\left(x_{1}, \cdots, x_{r-1}, v, x_{r+1}, \cdots, x_{p}\right) & \\
& -f\left(x_{1}, \cdots, x_{r-1}, u, x_{r+1}, \cdots, x_{p}\right) \mid,
\end{aligned}
$$

where $x_{1}, \cdots, x_{r-1}, x_{r+1}, \cdots, x_{p}, u, v$ vary in $[-1,1]$ with $0 \leqq v-u$ $\leqq \delta$. Let $n_{1}, n_{2}, \cdots, n_{p}$ be positive integers $\geqq 2$. Then

$$
\begin{gathered}
\sup _{\substack{-1 \leq x_{k} \leqq 1 \\
k=1,2, \cdots, p}}\left|f\left(x_{1}, x_{2}, \cdots, x_{p}\right)-H_{n_{1}, n_{2}, \cdots, n_{p}}\left(f, x_{1}, x_{2}, \cdots, x_{p}\right)\right| \\
\leqq \sum_{r=1}^{p}\left[2+4 \pi+\epsilon_{n_{r}}\right] \omega_{r}\left(n_{r}^{-1} \log n_{r}\right),
\end{gathered}
$$

where each $\epsilon_{n}(n=2,3, \cdots)$ depends on $n$ only, and $\epsilon_{n} \rightarrow 0$ as $n \rightarrow \infty$.

\section{REFERENCES}

1. L. Fejêr, Über Interpolation, Göttinger Nachrichten (1916), 66-91.

2. E. Moldovan, Observatii asupra unor procedee de interpolare generalizate, Acad. Repub. Pop. Romine, Bul. Şti. Sect. Ști. Mat. Fiz. 6 (1954), 477-482. (Romanian. French and Russian summaries)

3. O. Shisha, C. Sternin and M. Fekete, On the accuracy of approximation to given functions by certain interpolatory polynomials of given degree, Riveon Lematematika, 8 (1954), 59-64. (Hebrew. English summary)

\section{Aerospace Research Laboratories,}

Wright-Patterson Air Force Base, Ohio 